\title{
Abundances of Be Stars in Very Young Open Clusters
}

\author{
A. Marco, G. Bernabeu, J.M. Torrejón and J.J. Rodes \\ Departamento de Física, Ingeniería de Sistemas y Teoría de la Señal, \\ Universidad de Alicante, Ap.99, E-03080, Alicante, Spain
}

\section{J. Fabregat}

Departamento de Astronomía y Astrofísica, Universidad de Valencia, E-046100, Burjassot, Spain

\begin{abstract}
We present here standard $u v b y \beta$ CCD photometry for five open clusters of the Galaxy in order to determine abundances of Be stars, as a function of age.
\end{abstract}

\section{Introduction}

The long-term objective of this programme is the precise determination of abundances of Be stars, as a function of age, for very young open clusters. A chief part of this effort is to determine the ages of the clusters by means of isochrone fitting to the observational HR diagram, as well as precise determination of reddening and distance. In order to do this, we present here standard uvby $\beta$ CCD photometry for five open clusters of the Galaxy: NGC 1893, NGC 6910, NGC 884, NGC 869, NGC 2169.

\section{The observations}

The data were obtained with the JKT telescope (La Palma) in December 1997, using the $1024 \times 1024$ TEK \# 4 chip CCD and $u v b y \beta$ filters. Pixel size was 0.331 arcsec in such a way that the whole field covered by each frame was $5.6 \times 5.6$ arcmin. Three different observing times were scheduled for each filter and each cluster in order to reach the widest range of magnitudes. Standard stars were observed in the five clusters under study, using an intermediate exposure time.

\section{Reduction process}

The photometry has been obtained by PSF fitting using the DAOPHOT package (Stetson 1987) provided by IRAF, after bias and flat-field corrections. The atmospheric correction were perfomed using the RANBO2 program, based on the monochromatic extinction calculations of Manfroid (1993). Special care has been paid to the transformation to the standard system. A significative fraction of the observed stars in these clusters are highly reddened B type stars. Manfroid and Sterken (1987) and Crawford (1994) have shown that gross systematic errors 
may occur when the transformation process is applied to highly reddened stars, even if their colour range is fully bracketed by that of the standard stars. In order to avoid these effects we have elaborated a list of standards where only early type stars (MK earlier than A3, where the maximum of Balmer lines appears) are included, covering a wide range of reddening values.

\section{HR diagrams}

In the Figures we present $\mathrm{HR}$ diagrams for five clusters. The line represents the ZAMS given by Perry et al (1987). Values of reddening, distance modulus has been taken from the literature. The age has been calculated with the evolutionary models of Maeder and Meynet. As it can be seen, the photometry reaches very faint magnitudes, so that the whole $B$ main sequence is covered. We have catalogued a large number of new stars.
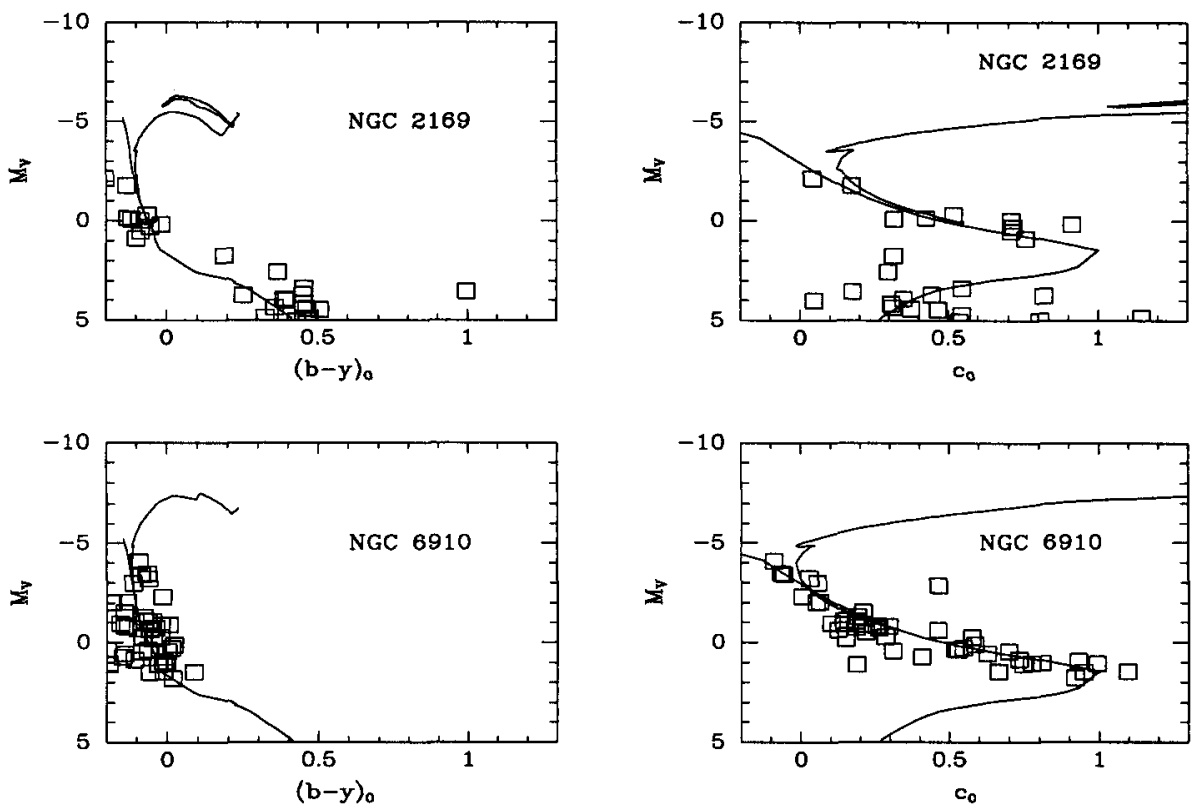

Figure 1. $M_{V}-(b-y)_{0}$ and $M_{V}-c_{0}$ diagrams for NGC 2169 and NGC 6910 .

\section{Abundance vs. Age}

One of the most striking features of young clusters is the differential reddening across cluster face which renders the calculus of the age quite uncertain. The exception is the Double Persei Cluster (NGC 884 and NGC 869), for which the observed isochrone has been very well fitted. It is found an age difference between the two clusters as well as a Be type star content difference: the older one (NGC 

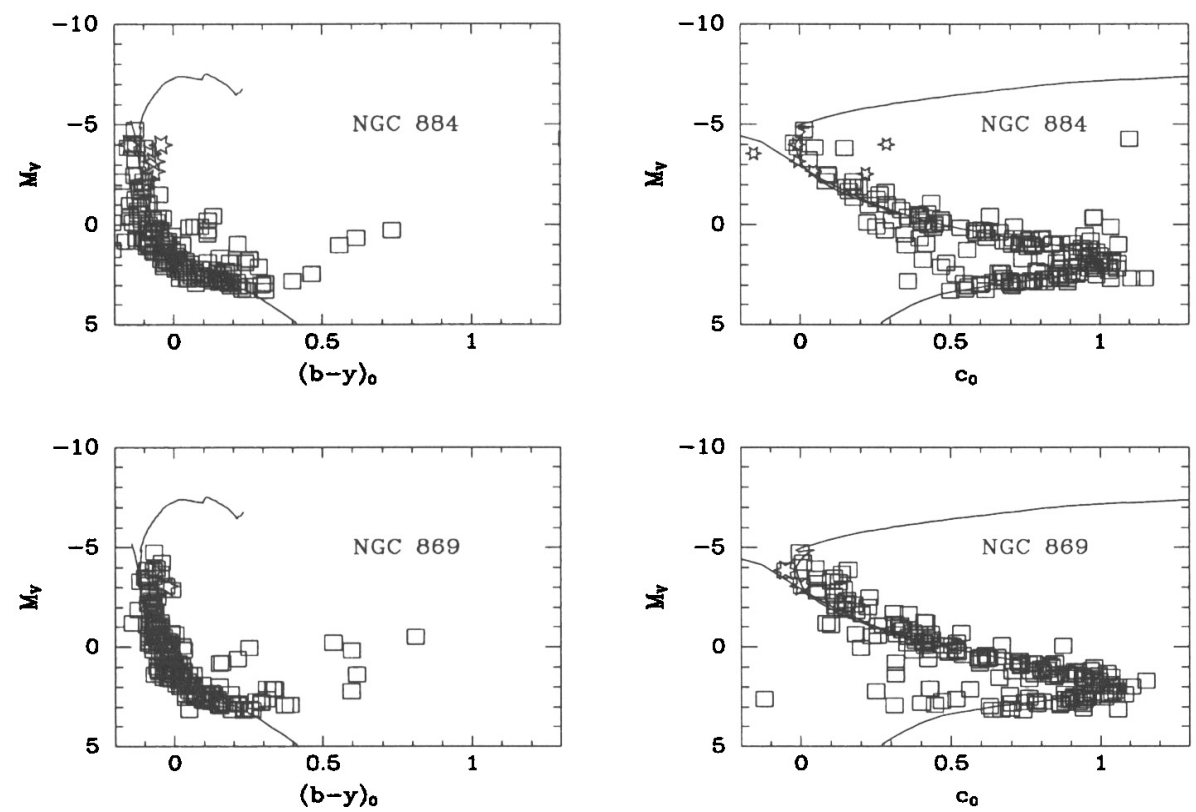

Figure 2. $M_{V}-(b-y)_{0}$ and $M_{V}-c_{0}$ diagrams for NGC 884 and NGC 869 . Be type stars are denoted by open stars.

$869, \log t=7.3$ ) has an fraction of Be type stars (all types, all luminosity classes) of $5-6 \%$ while the younger one (NGC $884, \log t=7.15$ ) has an abundance of $26 \%$. In NGC 1893 four new Be stars candidates has been found: 2-01-026, 1-02-23, 4-02-15 (nomenclature: Cuffey 1973) and 5001 (nomenclature: this work) on the grounds of their position at the HR diagrams and having $\beta$ indices which imply $\mathrm{H} \beta$ line in emission. All these stars have spectral types later than B5. Spectroscopy of these stars is needed to asses their true nature. In the rest of the clusters the presence of Be type stars with spectral type later than B5 is almost negligible. We present the relevant data in the Table 1:

Table 1. Abundance vs. Age for five open clusters

$\begin{array}{llcccc}\text { Cluster } & \log t & \mathrm{~N}_{\mathrm{Be}} & \text { New Be } & N_{\mathrm{B}+\mathrm{Be}} & \% \mathrm{Be} \\ \text { NGC 1893 } & 6.6 & 2 & 4 & 109 & 2-7 \\ \text { NGC 6910 } & 6.9 & 0 & 0 & 30 & 0 \\ \text { NGC 884 } & 7.15 & 6 & 0 & 23 & 26 \\ \text { NGC 869 } & 7.30 & 2 & 0 & 72 & 5-6 \\ \text { NGC 2169 } & 7.36 & 0 & 0 & 10 & 0\end{array}$

Even if the four emission line stars found in NGC 1893 were Be type stars, the abundance of Be stars.vs.age is not monotonic, but it seems to present a maximum around $14 \mathrm{Myr}$. 

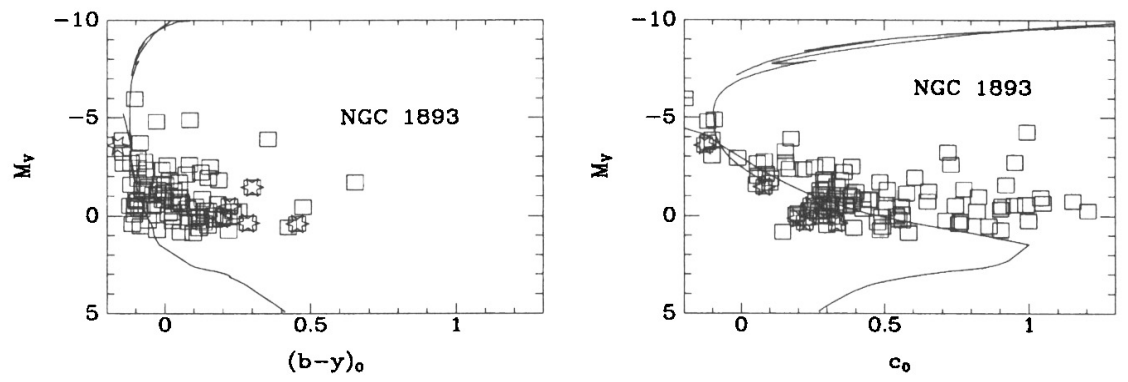

Figure 3. $M_{V}-(b-y)_{0}$ and $M_{V}-c_{0}$ diagrams for NGC 1893. Be type stars are denoted by open stars.

\section{References}

Crawford, D.L. 1994, PASP 106, 397

Manfroid, J. 1993, A\&A 271, 714

Manfroid, J. Sterken, C. 1987, A\&AS 71, 539

Perry, C.L. Olsen, E.H. Crawford, D.L. 1987, PASP 99, 1184

Stetson, P.B. 1987, PASP 99, 191

Cuffey, J. 1973, AJ 78, 747 